\title{
Diagnosis and treatment challenges of Sheehan's syndrome
}

\author{
Brittany E Gaspar* \\ University of North Florida, Jacksonville, USA
}

\begin{abstract}
Sheehan's Syndrome is a chronic health condition specific to women who have experienced postpartum hemorrhage. It is characterized by varying degrees of pituitary hormone imbalance related to the pathological process of pituitary gland necrosis after hemorrhage has occurred. Though the syndrome is a rare complication of hemorrhage, diagnosis is often substantially delayed due to the circumstances surrounding delivery and the broad spectrum of clinical presentation. The aim of this paper is to explore the complexities of the syndrome in an effort to help practitioners provide early diagnosis and appropriate treatment to women with the condition. This is achieved by synthesizing relevant and recent literature about immediate and delayed presentations of the condition via the frame of a clinical vignette.
\end{abstract}

According to the World Health Organization, roughly 14 million women experience postpartum hemorrhage every year [1]. Postpartum hemorrhage is defined as a total blood loss of $1,000 \mathrm{~mL}$ or more with associated hypovolemia within the first 24 hours after giving birth [2]. A severe hemorrhage is one in which blood loss exceeds $3,000 \mathrm{~mL}$ [3]. Postpartum hemorrhage can be fatal, but mortality from hemorrhage has been on the decline over the last 20 years. The decline in mortality has led to a rise in the recognition and diagnosis of a rare complication of postpartum hemorrhage known as Sheehan's Syndrome. Sheehan's Syndrome is a chronic health condition specific to women who have experienced postpartum hemorrhage characterized by varying degrees of pituitary hormone imbalance related to the pathological process of pituitary gland necrosis after hemorrhage has occurred. The condition is often low on the differential list due to vague symptomology that is largely determined by age, rapidity of onset, and the proportion of affected pituitary cells [4].

\section{Incidence and prevalence}

It is estimated that postpartum hemorrhage takes place in 1-2\% of all live births [5]. Sheehan's Syndrome occurs in 1 out of every 100,000 births globally and is the most common cause of hypopituitarism in underdeveloped or mid-income countries $[4,6]$. Lack of access to sophisticated medical procedure, skilled professionals, and medical resources contribute to higher rates of postpartum hemorrhage and subsequent Sheehan's Syndrome in developing nations, where the syndrome is said to occur in 5 out of every 100,000 births $[7,8]$. Incidence estimates vary greatly, with a range of $0-30 \%$ of cases reported following postpartum hemorrhage [9]. The disease is deemed "rare" in industrialized nations, but there has been an increase in prevalence of the disorder due to the flow of immigrants into these nations from developing countries, where the disorder is more prevalent [10]. It is reported that Sheehan's Syndrome accounts for $0.5 \%$ of all known cases of hypopituitarism in females [11].

Estimates of incidence and prevalence are relatively difficult to obtain due to the fact that diagnosis is commonly delayed due to the chronic nature of the condition. There have been varied reports on timeframe of diagnosis. One study reports that the average time between hemorrhage and diagnosis ranges from 1 to 33 years [12]. Another study found that the average time between hemorrhage and diagnosis for their study population was 2 to 40 years [6]. A study of the industrialized nation France found that the average time between hemorrhage and diagnosis was approximately 9 years [13]. Delayed diagnosis is attributed to the practitioners' inability to link broad spectrum symptomology to the condition. This inability does not stem from indifference, but rather stems from a lack of awareness of the rare condition and the lack of focus on postpartum and menstrual history in the years following delivery [14].

\section{Risk factors}

The primary risk factor for the development of Sheehan's Syndrome is pregnancy. The pathophysiologic changes of the pituitary gland in pregnancy leave the gland vulnerable to ischemia in the event of hemorrhage. Comparable blood loss in nonpregnant women does not cause hypopituitarism, lending credibility to this claim [3]. Postpartum hemorrhage can be difficult to predict due to the unpredictable nature of the birthing process. It is known that women that have experienced previous postpartum hemorrhage are three times more likely to have postpartum hemorrhage in their subsequent pregnancies [15]. One case analysis concluded that those diagnosed with Sheehan Syndrome have a significantly higher disseminated intravascular coagulation (DIC) score than their non-condition counterparts [4]. The DIC score, which is based upon patient disease history, clinical symptomology, and coagulation labs, may be an important determinant of potential for disease development [16]. Women with Sheehan's Syndrome report more pregnancy complications than their non-condition counterparts. The associated complications of stillbirth, miscarriage, abruption, placental retention, uterine rupture, hysterectomy, and pregnancy with multiples are all associated with potential for hemorrhage are reported more frequently and are known to cause potential life-threatening hemorrhage risks $[3,17]$.

${ }^{\star}$ Correspondence to: Brittany E. Gaspar, School of Nursing, University of North Florida, 1 UNF Drive, Jacksonville, FL 32224, FL 32205, USA, Tel: (352) 2835690, E-mail: brittanygasparbsn@gmail.com

Key words: sheehan's syndrome, postpartum hemorrhage, pituitary disorder Received: July 02, 2018; Accepted: July 23, 2018; Published: July 26, 2018 


\section{Pathophysiology}

Hyperplasia of the pituitary gland is a normal pathophysiologic process of pregnancy. This hyperplasia is attributed to the growth of prolactin-secreting lactotrophs, as they attempt to prepare the body for breastfeeding $[17,18]$. The pituitary gland increases in both volume and physical size, with an estimated $120-136 \%$ growth throughout the course of the pregnancy $[3,19]$. Increasing size correlates to increased nutritional and metabolic needs, but the arteries supplying blood to the gland remain unchanged [7]. The large pituitary gland compresses the superior hypophysial arteries, which supply the anterior pituitary with rapid, low-pressure flow [3]. The compression has minimal effect when the body is operating optimally [6].

After delivery, the gland enlarges slightly, but this hyperplasia stops approximately three days after delivery. The gland should return to prepregnancy size within six months in an uncomplicated pregnancy [20]. When postpartum hemorrhage occurs, the resultant hypotension and hypovolemic shock cause a decline in blood flow to the gland. Superior hypophysis compression impedes adequate perfusion throughout the hemorrhage process $[4,6]$. The resulting pituitary gland ischemia causes necrosis of the adenohypophysis, which can be further complicated by the hypercoagulable state of pregnancy [17]. The area of necrosis converts to scar tissue, causing the gland itself to atrophy. The result is physical destruction and dysfunction of the anterior pituitary lobe [4].

The pituitary gland tissue is not able to regenerate, meaning that these changes cannot be physically reversed $[3,18]$. The gland is still considered to be appropriately functioning if $50 \%$ or less is atrophied. Sheehan's Syndrome is considered in any presentation in which 70 $90 \%$ of the gland has experienced necrosis and subsequent scarring [4]. The severity of the condition is determined by how many hormones are affected by tissue destruction [6]. It is posited that tissue destruction continues to occur after the initial infarct. Necrosed tissue releases antigens, which promote the development of anti-pituitary antibodies. These antibodies grow in number over time, and they attack the remaining pituitary tissue. The percentage of available tissue dwindles, which causes increased hormone interruption and progression of presentation [3].

\section{Clinical presentation and considerations}

Consider the following case presentation: a 33-year-old Caucasian female presents to her primary care physician complaining of hair loss, cold intolerance, hypotension, extreme fatigue, and weight gain.

\section{Review of systems}

Common symptoms of Sheehan's Syndrome that may not present in the immediate postpartum include atrophy of the breasts, fatigue, decline in pubic, axillary, and facial hair (such as eyebrows), dryness or wrinkling of the skin, hypothyroid and associated symptomology (such as cold intolerance), chronic anemia, hypoglycemia, altered level of consciousness, infertility, hyponatremia, hypocortisolemia, and hypotension $[4,6,22]$. Depending on the severity of the damage to the pituitary, these symptoms may present consistently, or may only present when the patient reports physical or psychological stress [13]. It is important to complete a detailed review of systems to assess for the potential of multiple symptoms existing or interacting at the same time. Often times, these symptoms are treated individually, rather than as a whole part of a single condition (Table 1).

\section{Past medical history}

The female patient states that this is her third attempt to seek resolution to this problem. She initially sought care for amenorrhea following her second delivery. The first doctor started her on estradiol/ progesterone tablets. The second doctor gave her a diagnosis of postpartum depression after she sought care for anxiety, tachycardia, and feelings of stress, but medications did not help symptoms. She tapered off of antidepressants and did not return. She reports no other medical history or diagnoses.

Because of variable symptomology and delayed diagnosis, it is important to inquire about pregnancy histories well beyond the postpartum period. In one study of Sheehan's patients, $52.6 \%$ of women with the condition reported previous hospitalizations for symptomology, but none had ever received the appropriate diagnosis [10]. Routinely asking about the details surrounding delivery locations, postpartum hemorrhage, hospitalizations, blood transfusions, and breastfeeding can provide important clues in working toward a differential of the disorder [6,11].

\section{Pregnancy and postpartum history}

G2P2. First delivery was a vaginal live birth with no complication. Second delivery was an emergency cesarean section at age 28 after failure to progress and fetal distress. The cesarean itself was without complication. Postpartum hemorrhage occurred after closing of surgical incision after the physician attempted fundal massage. Estimated blood loss was $4,000 \mathrm{~mL}$. Reports being told by the staff that

Table 1. Review of Systems [3,14,17,21]

\begin{tabular}{|l|l|l|}
\hline Symptom & Presence in Case Presentation & Meaning \\
\hline Hair Loss & + & Potential indicator of adrenal insufficiency \\
\hline Lactation abnormality & Not presently & Only present on ROS if within one year of delivery \\
\hline Hot Flashes & - & $\begin{array}{l}\text { Positive can be indicator of growth hormone imbalance or a normal finding in } \\
\text { postoperative hysterectomy }\end{array}$ \\
\hline Cold Intolerance & + & Potential indicator of secondary hypothyroidism \\
\hline Mood Disturbances & + & \\
\hline Weakness & + & Potential indicator of secondary hypothyroidism \\
\hline Extreme Fatigue & + & Potential indicator of secondary hypothyroidism and growth hormone imbalance \\
\hline Anorexia & - & \\
\hline Nausea & - & \\
\hline Stress & + & Stress can "activate" a latent Sheehan's Syndrome \\
\hline Mental Apathy & + & \\
\hline Weight Gain/Loss & + gain 30 lbs over 5 months & Potential indicator of growth hormone imbalance \\
\hline Decreased Libido & + & \\
\hline Syncope/Dizziness & + with home BP readings resulting SBP ranging from $80-110$ & \\
\hline
\end{tabular}


"the blood was coming out as fast as it was going in." No hysterectomy performed. Spent three weeks in the ICU. Healthy baby girl was bottlefed due to mother's ICU status. Amenorrhea after delivery reported.

The initial symptoms of Sheehan's Syndrome can be recognized in the immediate postpartum period. The two most immediate signs of Sheehan's syndrome are failure to lactate and menstrual irregularities [21]. Women with the syndrome are often unable to breastfeed due to a decline of milk production and secretion. This is evidenced in research, with one study reporting that $70 \%$ of the study sample did not breastfeed [6]. The largest barrier to missing this diagnostic sign is the protocol of placing women who have experience postpartum hemorrhage in the ICU, where breastfeeding is not allowed or simply not feasible [13]. Women and providers often think that milk production has declined due to the lack of stimulation from the act of breastfeeding, when in actually they have an underlying pathophysiologic condition. It is imperative to assess all patients who have experienced postpartum hemorrhage for milk production or breastfeeding concerns.

The most common menstrual abnormality in Sheehan's Syndrome is amenorrhea. Women with this syndrome do not begin menstruating within 6 months of giving birth, or if they do, the periods are irregular due to hormone imbalances. This aspect of the condition can often times be overlooked because of the fact that emergent hysterectomies are performed to treat unstable postpartum hemorrhage [13]. Many women speak with their providers before delivering about the resumption of birth control after delivery. Resuming the birth control before observing for signs of amenorrhea in someone with a postpartum hemorrhage history may mask this symptom [21].

\section{Objective data}

The patient exhibits many of the classic physical exam elements with the exception of breast atrophy and her normal Glasgow Coma Score. This score has been found to be significantly lower in Sheehan's cases when compared to non-Sheehan's cases in current research cases [4] (Table 2).

True Sheehan's Syndrome is defined as total hypopituitarism, in which all six hormones released by the anterior pituitary are affected. This means that there is permanent secretion dysfunction of growth hormone $(\mathrm{GH})$, followed by prolactin (PRL), follicle-stimulating hormone (FSH), luteinizing hormone $(\mathrm{LH})$, adrenocortiocotropic hormone (ACTH), and thyroid stimulating hormone (TSH). Serum estrogen (E2), IGF-1, free T4, and cortisol are also affected [4,7] (Table 3).

\section{Imaging}

The gold standard imaging for the diagnosis of Sheehan's Syndrome is MRI. The pituitary gland is housed in the sella turcica, which is a groove in the sphenoid bone. Pituitary gland necrosis empties the sella turcica. Imaging can reveal partial or full loss of the gland. Approximately $70 \%$ of all Sheehan's cases evidence a completely empty sella turcica $[8,11]$. CT scans can also help in diagnosis (Figure 1).

\section{Diagnosis}

Diagnosis of Sheehan's Syndrome requires synthesis of subjective and objective data points. Differentials commonly associated with the condition are hypothyroidism, postpartum depression, major depressive disorder, and pituitary tumor. Tumor can be ruled out via diagnostic imaging, which would evidence growth or invasion of other regions, or potential spontaneous resolution [3]. Major depressive disorder is associated with growth hormone deficiency.
Growth hormone is the first hormone to be affected in Sheehan's Syndrome. Treating depression alone will not help with symptom resolution. Depression can be ruled as a primary diagnosis based upon patient history and symptom progression [11]. Similarly, treating hypothyroidism in these patients will not help with overall symptom resolution. A detailed pregnancy and postpartum history should be taken for any female patient with suspected hypothyroid based on laboratory studies and subjective presentation (Table 4).

\section{Treatment}

Effective treatment of Sheehan's Syndrome includes treating every hormone irregularity present. Along with correcting these imbalances, consideration must be taken regarding the side effects and long-term implications of hormone therapy on the various body systems.
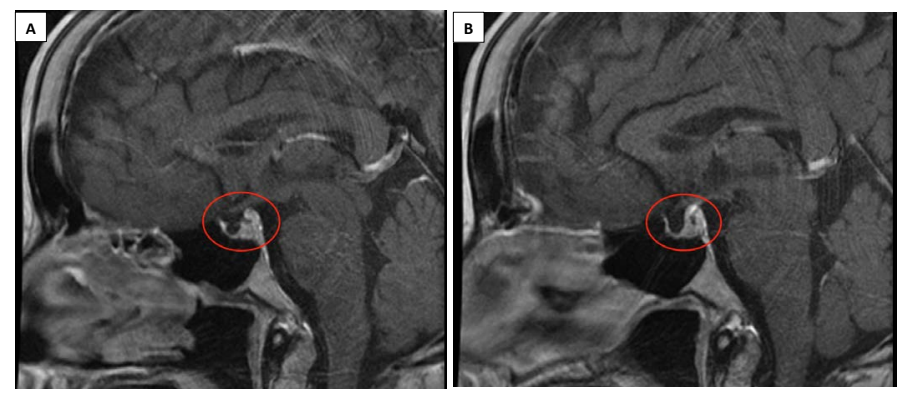

Figures 1. MRI imaging evidencing diminished pituitary size

Table 2. Noted Physical Findings

\begin{tabular}{|l|l|}
\hline Physical Finding & Presence in Case Presentation \\
\hline Breast Atrophy & Negative \\
\hline Hair Loss & $\begin{array}{l}\text { Positive: eyebrow, scalp, axilla, pubic area. } \\
\text { Remaining hair is coarse, fine }\end{array}$ \\
\hline Pallor & Pale \\
\hline Skin & Coarse, dry skin. Cool to touch. \\
\hline Glasgow Coma Scale & Normal score \\
\hline BMI/Weight Distribution & Obese BMI; abdominal girth \\
\hline Mood & Anxious \\
\hline
\end{tabular}

Table 3. Expected Laboratory Findings for Patient with Sheehan's Syndrome [3,4]

\begin{tabular}{|c|c|}
\hline Lab & Finding \\
\hline GH & Decreased \\
\hline PRL & Decreased \\
\hline FSH & Decreased \\
\hline LH & Decreased \\
\hline ACTH & Normal or elevated \\
\hline TSH & Normal or elevated \\
\hline E2 (serum) & Undetectable \\
\hline IGF-1 & Decreased \\
\hline Free T4 & Decreased \\
\hline Cortisol & Decreased \\
\hline
\end{tabular}

Table 4. Diagnostic Criteria [3]

Essential Diagnostic Criteria

History of Postpartum Hemorrhage

Accompanying hypotension, shock, or DIC requiring blood transfusions or fluid management

Inability to lactate after childbirth

Menstrual irregularity after that did not resolve spontaneously

At least one hormone deficiency from the anterior pituitary lobe

Empty or severely damaged sella turcica on diagnostic imaging 
Women with Sheehan's Syndrome are at an increased risk for osteoporosis related to hypogonadism. It is imperative that these women complete hormone replacement therapy in order to combat bone loss [6]. Oral contraceptives, such as ethinyl estradiol, and transdermal patches have both been proven successful for this purpose [23]. These women should have bone density testing and should be monitored closely. Education should be provided on appropriate weight-bearing exercise, the importance of routine monitoring, and the side effects of prolonged contraceptive use, especially the risk for blood clots.

The treatment of ACTH and thyroid imbalances are considered to be the most important aspects of treating Sheehan Syndrome. Treatment of ACTH is achieved with either hydrocortisone or prednisone. The dosage and selection of medication depends on the severity of the imbalance. Some patients need medications only when enduring stress, while others need medications consistently in order to maintain a baseline level [14]. If cortisol levels improve, patients may remain on small doses, increasing the frequency as appropriate $[4,23]$. Caution is recommended when using these medications, as Sheehan's Syndrome may cause an increase in neural receptors to steroids, leading to psychosis [24]. Corticosteroids may need to be coupled with an antipsychotic in the initial stages until cortisol regulation is achieved. Corticosteroid's should always be given before initiating thyroid treatment, as treatment of the thyroid alone can cause adrenal crisis to occur [3]. Free T4 imbalance is commonly treated with Synthroid.

GH must be replaced if there are three or more known deficient pituitary hormones [3]. Replacement is achieved via self-injection. Females must be monitored closely while receiving injections, as they classically do not respond as effectively as males and may require higher doses [23]. It is important to education the patient that these therapies are all long-term. For GH injections, proper technique must be instructed, as the injections will not be as effective if they are improperly administered. The psychosocial and environmental situations of the patient must also be considered prior to prescribing $\mathrm{GH}$ injections due to the nature of the medication.

Routine monitoring is the only way to ensure adherence to the regimen and effective disease management. The patient assumes a large amount of responsibility. The patient should be referred to case management to help promote disease management and ensure that appropriate medications can be attained. Frequent trips to the office and to the lab must be secured, and a referral to endocrinology is best to ensure long-term compliance and management.

\section{Prevention and management: Moving forward}

There are no preventative measures in place to stop the development of Sheehan's Syndrome following postpartum hemorrhage. Some researchers hypothesize that transfusing close to the time of hemorrhage acts as an immediate intervention, but there is no definitive evidence for this. The disease is progressive in nature if left untreated, as the body develops antibodies to the dead pituitary tissues. These antibodies attack the tissue, which can cause worsening imbalances over time [10]. The only way to slow the progression and severity of presentation is early diagnosis and effective management. As providers, we can help facilitate diagnosis and treatment simply by listening. Listen to your patient's history. Ask them about their pregnancies, even if their pregnancies were over ten or even twenty years ago. Consider this history when completing your review of systems, and order labs preemptively based on history. For providers caring for patients in the immediate postpartum after hemorrhage, implement a screening tool that assesses for the early signs of amenorrhea and inability to breast feed. In order to effectively manage this rare condition, adapt a proactive, rather than reactive, approach.

\section{Authorship}

I, Brittany Gaspar, am the author of this submission, and have adhered to all editorial policies for submission as described on the resources available on the OAT website. I attest to having met all authorship criteria.

\section{Acknowledgements}

The author would like to acknowledge Kimberly Gaspar for providing MRI imaging and personal experiences that helped shape the clinical case presentation within this manuscript.

\section{References}

1. World Health Organization (2013) Priority diseases and reasons for inclusion [Available at: http://www.who.int/medicines/areas/priority_medicines/Ch6_16PPH. pdf]

2. American College of Obstetricians and Gynecologists (2017) ACOG practice bulletin: Clinical management guidelines for obstetrician-gynecologists: Postpartum hemorrhage (Number 183). [Available at: http://clinicalinnovations.com/wp-content/ uploads/2017/10/ACOG_Practice_Bulletin_No_183_Postpartum-Hemorrhage-2017. pdf]

3. Diri H, Karaca Z, Tanriverdi F, Unluhizarci K, Kelestimur F (2016) Sheehan's syndrome: new insights into an old disease. Endocrine 51: 22-31. [Crossref]

4. Matsuwaki T, Khan KN, Inoue T, Yoshida A, Masuzaki H (2014) Evaluation of obstetrical factors related to Sheehan syndrome. J Obstet Gynaecol Res 40: 46-52. [Crossref]

5. Gibbons KJ, Albright CM, Rouse DJ (2013) Postpartum hemorrhage in the developed world: Whither misoprostol? Am J Obstet Gynecol 208: 181-183.

6. Dokmetas HS, Kilicli F, Korkmaz S, Yonem O (2006) Characteristic features of 20 patients with Sheehan's syndrome. Gynecol Endocrinol 22: 279-283. [Crossref]

7. Schury MP, Adigun R (2017) Sheehan syndrome. StatPearls. Treasure Island (FL): StatPearls Publishing.

8. Shivaprasad C (2011) Sheehan's syndrome: Newer advances. Indian J Endocrinol Metab 15 Suppl 3: S203-207. [Crossref]

9. González-González JG, Borjas-Almaguer OD, Salcido-Montenegro A, RodríguezGuajardo R, et al. (2018) Sheehan's Syndrome Revisited: Underlying Autoimmunity or Hypoperfusion? Int J Endocrinol 2018: 8415860. [Crossref]

10. Diri H, Tanriverdi F, Karaca Z, Senol S, Unluhizarci K, et al. (2014) Extensive investigation of 114 patients with Sheehan's syndrome: a continuing disorder. Eur $J$ Endocrinol 171: 311-318. [Crossref]

11. Qadri MI, Mushtaq MB, Qazi I, Yousuf S, Rashid A (2015) Sheehan's Syndrome Presenting as Major Depressive Disorder. Iran J Med Sci 40: 73-76. [Crossref]

12. Huang YY, Ting MK, Hsu BR, Tsai JS (2000) Demonstration of reserved anterior pituitary function among patients with amenorrhea after postpartum hemorrhage. Gynecol Endocrinol 14: 99-104. [Crossref]

13. Ramiandrasoa C, Castinetti F, Raingeard I, Fenichel P, Chabre O, et al. (2013) Delayed diagnosis of Sheehan's syndrome in a developed country: A retrospective cohort study. Eur J Endocrinol 169: 431-438.

14. Thyagaraj V, Kumar MJ (2015) Diagnosis Delayed but not Denied - Sheehan's syndrome. JNMA J Nepal Med Assoc 53: 31-33. [Crossref]

15. Oberg AS, Hernandez-Diaz S, Palmsten K, Almqvist C, Bateman BT (2014) Patterns of recurrence of postpartum hemorrhage in a large population-based cohort. Am J Obstet Gynecol 210: 229. [Crossref]

16. Kobayashi T (2014) Obstetrical disseminated intravascular coagulation score. J Obstet Gynaecol Res 40: 1500-1506.

17. Soni M (2014) Sheehan syndrome: A rare complication of post-partum hemorrhage. Nepal J Obstet Gynaecol 18: 61-63.

18. Kovacs K (2003) Sheehan syndrome. Lancet 361: 520-522. [Crossref] 
19. Gonzalez JG, Elizondo G, Saldivar D, Nanez H, Todd LE, et al. (1988) Pituitary gland growth during normal pregnancy: An in vivo study using magnetic resonance imaging. Am J Med 85: 217-220.

20. Dinç H, Esen F, Demirci A, Sari A, Resit Gümele H (1998) Pituitary dimensions and volume measurements in pregnancy and post partum. MR assessment. Acta Radiol 39: 64-69. [Crossref]

21. Tovar YE, Ahmed W, Urella M, Elkadry AH, Khthir RA (2017) Sheehan's syndrome: It is time to become more proactive in identifying this disease. Marshall J Med 3: 27-33.
22. Hao J, Liu M, Mo Z (2012) The Symptoms Get Worse after Pregnancy in Sheehan's Syndrome: A Case Report. Case Rep Med 2012: 271345. [Crossref]

23. Kim SY (2015) Diagnosis and Treatment of Hypopituitarism. Endocrinol Metab (Seoul) 30: 443-455. [Crossref]

24. Chatterjee SS, Mitra S, Mallik N, Ghosal M (2016) Even very low dose hydrocortisone might precipitate psychosis in Sheehan's syndrome: A need for caution. $J$ Neurosci Rural Pract 7: 333-334. [Crossref]

Copyright: (C2018 Gaspar BE. This is an open-access article distributed under the terms of the Creative Commons Attribution License, which permits unrestricted use, distribution, and reproduction in any medium, provided the original author and source are credited. 